\title{
Congenital Laryngeal Cyst in Newborn
}

\author{
Mariya Zakharova* and Pavel Pavlov
}

Russia

ISSN: 2576-9200

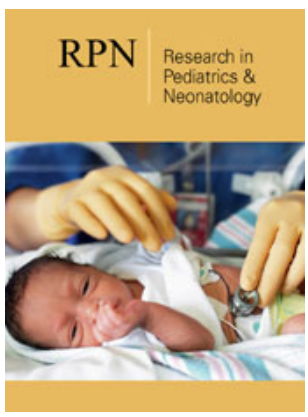

${ }^{* 1}$ Corresponding

author:

Mariya

Zakharova, Russia

Submission: 眥 April 08, 2019

Published: 制 May 17, 2019

Volume 3 - Issue 3

How to cite this article: Zakharova $M$, Pavlov P. Congenital Laryngeal Cyst in Newborn. Res Pediatr Neonatol. 10(2). RPN.000565.2019.

DOI: $10.31031 / R P N .2019 .03 .000565$

Copyright@ Mariya Zakharova, This article is distributed under the terms of the Creative Commons Attribution 4.0 International License, which permits unrestricted use and redistribution provided that the original author and source are credited.

\section{Abstract}

Case of congenital laryngeal cyst in 11 month's children, successfully treated in ENT department SPSPMU. Diagnostic and optimal surgical tactic for patients with cystic laryngeal dysplasia are described.

Keywords: Congenital laryngeal cyst; Cystic laryngeal dysplasia; Congenital abnormalities of the larynx

\section{Case Report}

In this article, we bring to your attention a clinical observation of the successful surgical treatment of congenital laryngeal cysts in an infant. Girl B, 11 months in September 2013, was routinely admitted to the otolaryngology department of SPbPMU with complaints about the impossibility of breathing through the natural respiratory tract, the presence of a tracheostomy.

From the anamnesis: a child from 1 pregnancy, proceeding with a pathological weight gain. Births first, in terms of 38-39 weeks, elective caesarean section. The birth weight is $2800 \mathrm{~g}$, height $47 \mathrm{~cm}$. In connection with a breathing disorder from the first minutes of life, the girl was transferred to intensive care, where she was intubated. Intubation was carried out for a long time with technical difficulties. The girl was transferred to a clinical hospital, where she was on prolonged intubation and after unsuccessful attempts at extubating, a tracheostomy was applied for 21 days of life. Discharged from the hospital at the age of 1 month 14 days, Diagnosis: cerebral ischemia 2 tablespoons, depression syndrome. Natal trauma of the cervical spine. Post-traumatic laryngitis. Stenosis of the larynx 3tbsp. Fetal infection of unspecified etiology (pneumonia, DN 2st). Syndrome of acute renal failure. Broncho-pulmonary dysplasia of moderate severity. Heart rhythm disturbance-supraventricular extrasystole. Open oval window. NK 0. Anemia of moderate severity, mixed genesis. IUGR of hypertrophic type $1 \mathrm{tbsp}$.

Observed by a pediatrician, otorhinolaryngologic, neurologist at the place of residence. Diagnosis at the time of admission to SPbPMU: Cicatricial stenosis of the larynx. Congenital malformation of cartilage of the larynx. Paresis of the right vocal fold. Tracheostomy. On admission: satisfactory condition, breathing through a tracheostomy, endoscopy of the nasal cavity, pharynx, otoscopy without signs of inflammation. Aphonia.

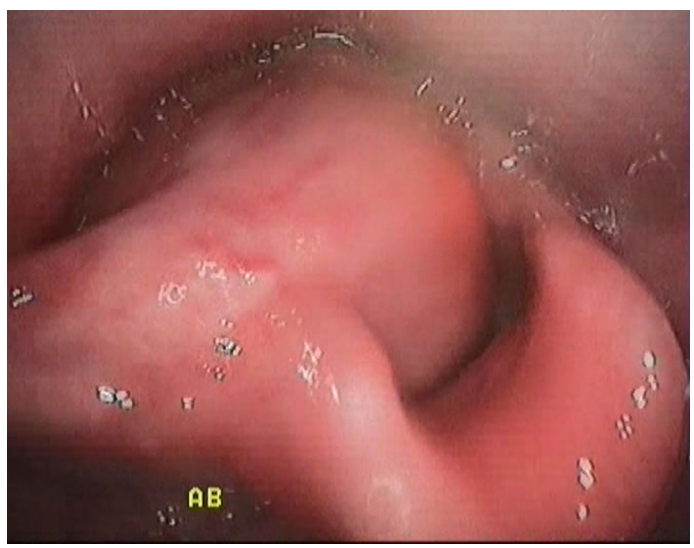

Figure 1: Fibro video laryngoscopy. In the area of the right gravel of the fold, a rounded formation is visualized, which almost completely covers the lumen of the larynx. 
When fibro laryngoscopy revealed a rounded formation of the right vysokopalonadgortnaya fold almost completely overlapping the lumen of the larynx (Figure 1). On a series of MRI tomograms of the soft tissues of the neck from the level of C2 to the level of C5-6 in the projection of the right pear-shaped sinus is determined by a cyst with clear uneven contours, which has an irregular rounded shape. The size of the cyst is $11 \times 16 \times 28 \mathrm{~mm}$. Cyst significantly shifts the right scooped-nadgortnaya fold to the left. Its lower edge is traced at the level of the subglottic space (Figure 2-4).

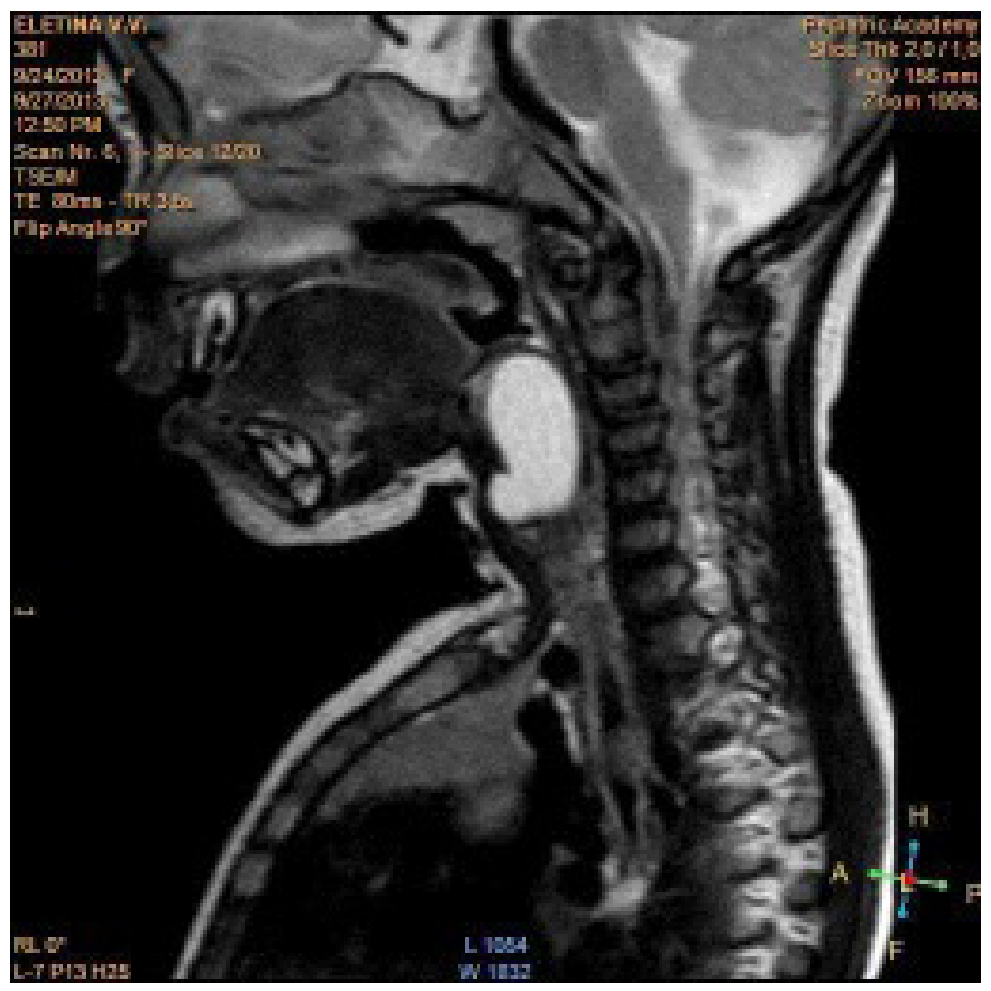

Figure 2: MRI, sagittal projection.

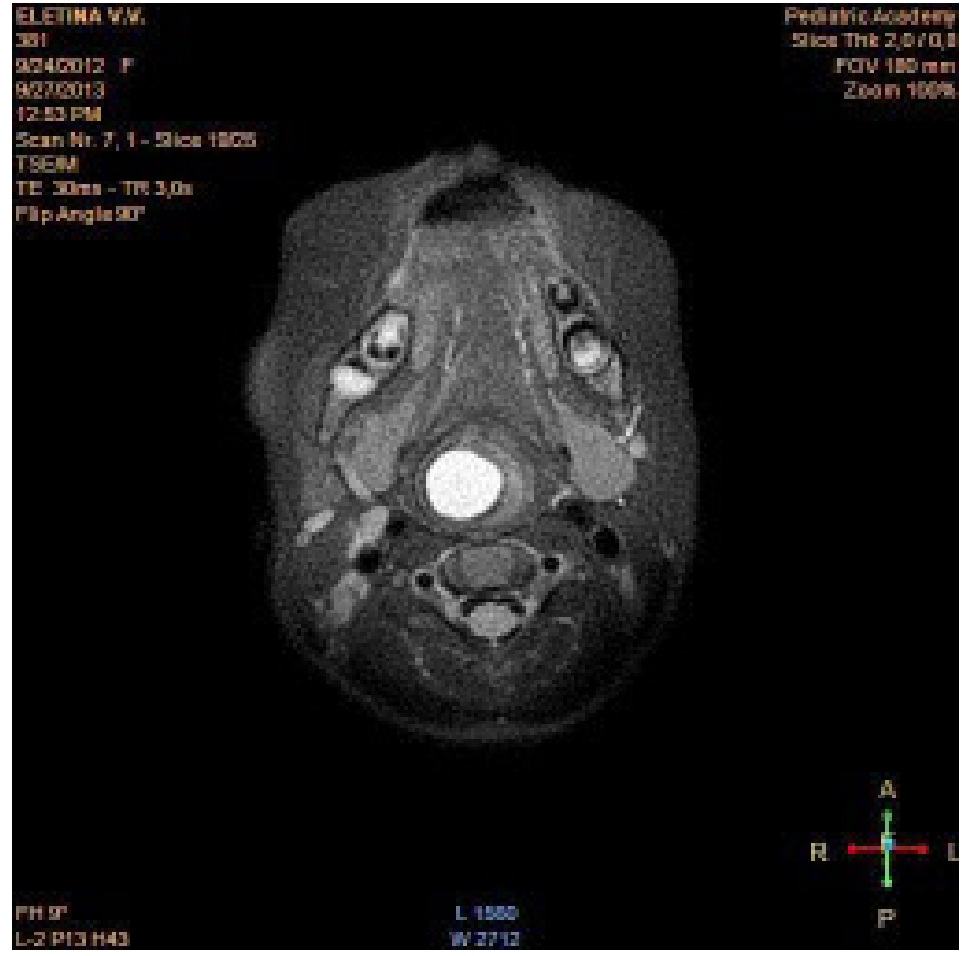

Figure 3: MRI, coronary projection. 


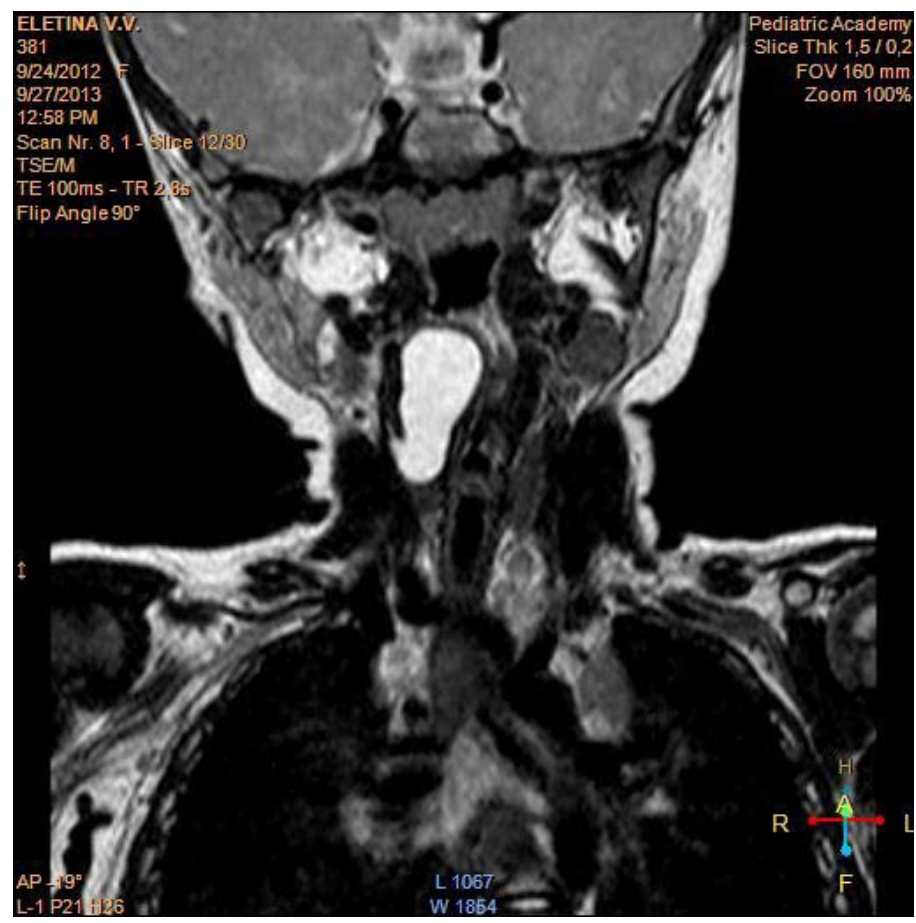

Figure 4: MRI, frontal projection.

Adirect suspended micro video laryngoscopy with simultaneous endoscopic marsupialization of the cyst was performed. Under the control of the operating microscope, the Atkus-15diode laser in the contact mode power of $5 \mathrm{~W}$, a cyst wall incision was made, parallel to the crayon-donor fold. About $7 \mathrm{ml}$ of a thick gel-like secret was aspirated from the cyst, after emptying we began to observe the voice section. An excision of the cyst wall section, medial to the incision line, was performed.

Diagnosis of cysts of this localization is not difficult if there is a possibility of fibro laryngoscopy, MRI or MSCT at the disposal of doctors, which will allow to estimate the prevalence of a cyst and carry out its differential diagnosis with other laryngopharyngeal neoplasms. The final stage of diagnosis is direct micro videoholingoscopy, which can be combined with surgical treatment.

\section{Conclusion}

According to the literature, endoscopic marsupialization of a cyst using a contact laser or a microtool is the method of choice for surgical treatment of such formations. This method is the least traumatic and does not give a relapse. In our case, performing endoscopic marsupialization with the help of a contact laser allowed us to decannulate the child. 